\title{
ON STOP-LOSS ORDER AND THE DISTORTION PRICING PRINCIPLE
}

\author{
By WERNER HÜRLIMANN,
}

Winterthur

\begin{abstract}
A number of more or less well-known, but quite complex, characterizations of stop-loss order are reviewed and proved in an elementary way. Two recent proofs of the stop-loss order preserving property for the distortion pricing principle are invalidated through a simple counterexample. A new proof is presented. It is based on the important Hardy-Littlewood transform, which is known to characterize the stop-loss order by reduction to the usual stochastic order, and the dangerousness characterization of stop-loss order under a finite crossing condition. Finally, we complete and summarize the main properties of the distortion pricing principle.
\end{abstract}

KEY WORDS

Pricing theory, distortion function, quantile function, stop-loss order, stochastic order, Hardy-Littlewood transform

\section{INTRODUCTION}

Since its introduction by Bühlmann (1970), the functional approach to premium calculation in insurance has seen an impressive development. A first general and rather elementary method to generate valuable pricing principles consists of the class of quantile premium calculation principles by Denneberg (1985/90/94). Several recent contributions around this theme have been made in actuarial science and finance, among others Hürlimann (1993), Wang (1995a/b/c, 1996a/b), Wang et al. (1997) and Chateauneuf et al. (1996).

For a given set $\mathrm{S}$ of non-negative random variables $X \geq 0$ with finite means, defined on some probability space, and which represent random losses of insurance contracts, a pricing principle is a non-negative real function $P: S \rightarrow R$, which depends on the distribution $F_{x}(x)$ of $X$, and which is interpreted as price of the insurance risk. From an axiomatic point of view, it is well accepted that a pricing principle should satisfy a certain 
number of desirable properties. Without repeating all well-known interpretations, the following properties are quite reasonable:

(P1) $\quad P[X] \geq E[X]$, for all $X \in S$

(P2) $\quad P[X] \leq \sup [X]$, for all $X \in S$

(P3) $\quad P[a X+b]=a P[X]+b$, for all $a, b, a>0$, for all $X \in S$

(P4) $\quad P[X+Y] \leq P[X]+P[Y]$, for all $X, Y \in S$ such that $X+Y \in S$

(P5) $\quad P[X] \leq P[Y]$ if $X \leq_{s l} Y$ and $X, Y \in S$

The last property says that the price functional preserves the stop-loss order, or equivalently the increasing convex order (see Kaas et al. (1994) and Shaked and Shanthikumar (1994) for fundamentals). Requiring that the price functional preserves the usual stochastic order $\leq_{s t}$ only, is a less stringent property since stochastic order implies stop-loss order. Though the stop-loss ordering preserving property of the Swiss family of premium calculation principles has been known since its actuarial consideration in Bühlmann et al. (1977), the recognition of $\leq_{s l}$ as a sound ordering of risk seems more recent. For example, the order preserving axiom $(P 5)$ is considered in Heilmann (1987) but without mention of a specific and accepted partial order, which could be used as selected ordering of risk. Furthermore, the absolute deviation principle and the Gini principle, introduced by Denneberg (1985/90), and which satisfy properties $(P 1)-(P 4)$, and the weaker stochastic order preserving property, also satisfy $(P 5)$, (consequence of our main result in Section 3.2). Previously two quite similar but different proofs of (P5) have been proposed by Wang $(1996 \mathrm{a} / \mathrm{b})$, but both contain an error (see Section 3.1).

In view of the above discussion, it seems useful to present a short chronological review of some main non-trivial pricing functions, which preserve $\leq_{s l}$, and inspect whether the remaining axioms $(P 1)-(P 4)$ are satisfied.

The Swiss family is positively homogeneous if, and only if, it is the net principle (see Schmidt (1989), simpler proof by Hürlimann (1997), Example 4.1 (continued), p. 9). The first genuine pricing principles, which satisfy (P1)-(P5), are the absolute deviation principle $P[X]=E[X]+\theta \cdot E\left[\left|X-m_{X}\right|\right], 0 \leq \theta \leq 1$ (Denneberg $(1985 / 90)$ ) and the Gini principle $P[X]=E[X]+\theta \cdot \operatorname{Gini}[X]$, $0 \leq \theta \leq 1$ (Denneberg (1990)). These functionals are special cases of the class of distortion pricing principles.

$$
P[X]=\int_{0}^{\infty} g\left(\bar{F}_{X}(x)\right) d x=\int_{0}^{1} F_{X}^{-1}(1-u) d g(u)=\int_{0}^{1} F_{X}^{-1}(u) d \gamma(u),
$$

where $g(x)$ is an increasing concave function such that $g(0)=0, g(1)=1$, $\bar{F}_{X}(x)=1-F_{X}(x)$ is the survival function, $\gamma(x)=1-g(1-x)$ is the distortion of probabilities in Denneberg's setting, and $F_{X}^{-1}(u)$ is a quantile function of $X$. The second equality is obtained through partial integration, 
and shown by elementary calculus in case $g(x)$ is differentiable. The righthand side representation has been introduced by Denneberg (1990) and its equivalence with the first integral (up to an alternative appropriate definition of the inverse) has been used by Wang (1996a) (see also Wang et al. (1997)).

Another attractive special case of (1.1) is the PH-transform principle studied by Wang (1995a/95b/96a/96b). Previously to the last example had appeared the Dutch principle (see van Heerwaarden (1991), van Heerwaarden and Kaas (1992), Kaas et al. (1994) and a slight generalization of it (see Hürlimann (1994/95a/95b)). A pricing principle from the Dutch family satisfies (P1)-(P5) if, and only if, it is of the form

$$
P[X]=E[X]+\theta \cdot E\left[(X-E[X])_{+}\right], \quad 0 \leq \theta \leq 1 .
$$

The Dutch family is a special case of the class of so-called "quasi-mean value principles" considered recently by the author. However, only sporadic members of this class define feasible price functionals satisfying (P1)-(P5), of which one may mention the interesting Example 11.1 in Hürlimann (1997a).

A generalization of the class of distortion pricing principles is the class of Choquet pricing principles in Chateauneuf et al. (1996), which is based on the theory of capacities and non-additive measures (exposed in Denneberg (1994)), and breaks with the traditional probabilistic foundations of actuarial science and finance. Finally, let us mention that one misses still feasible price functionals along the economic approach initiated by Bühlmann (1980/84) (see the critical comments by Lemaire (1988)).

In the present paper, we invalidate Wang's proofs of the property $(P 5)$ for the distortion pricing principle through a simple counterexample, and focus on a new proof of this important property. Using a two-stage limiting argument (dominated convergence theorem and continuity property of the distortion pricing functional), it is possible to restrict the attention to risks, which belong to the following large set:

$\mathrm{S}$ consists of all non-negative random variables with finite means, such that the distribution functions of any two of them cross finitely many times (finite crossing condition).

For completeness, we show also that (1.1) satisfies the other properties $(P 1)-(P 4)$, where our expose is intended to be essentially accessible from an elementary perspective.

The paper is organized as follows. In Section 2, a number of more or less well-known, but quite complex, characterizations of stop-loss order are reviewed and proved in an elementary way. Since no such proofs have been found in the original and other papers (and books) consulted by the author, the present supplement to the existing literature will hopefully be helpful for future workers in this area (as it has been to the author). Section 3 is devoted to a derivation of the main properties of the distortion pricing principle. In Section 3.1 two recent proofs by Wang of the stop-loss order preserving property for the distortion pricing principle are invalidated through a simple 
counterexample. A new proof is presented in Section 3.2. It is based on the important Hardy-Littlewood transform, which is known to characterize the stop-loss order by reduction to the usual stochastic order (Theorem 2.3), and the dangerousness characterization of stop-loss order under the finite crossing condition (1.3) (Theorem 2.2). Finally, we complete and summarize the main properties of the distortion pricing principle in Section 3.3.

\section{SOME EQUIVALENT CHARACTERIZATIONS OF STOP-LOSS ORDER}

Capital letters $X, Y, \ldots$ denote random variables with distribution functions $F_{X}(x), F_{Y}(x), \ldots$ and finite means $\mu_{X}, \mu_{Y}, \ldots$. The survival functions are denoted by $\bar{F}_{X}(x)=1-F_{X}(x), \ldots$. The stop-loss transform of a random variable $X$ is defined by

$$
\pi_{X}(x):=E\left[(X-x)_{+}\right]=\int_{x}^{\infty} \bar{F}_{X}(t) d t, \quad x \text { in the support of } X .
$$

The random variable $X$ is said to precede $Y$ in stochastic order or stochastic dominance of first order, a relation written as $X \leq_{s t} Y$, if $\bar{F}_{X}(x) \leq \bar{F}_{Y}(x)$ for all $\mathrm{x}$ in the common support of $X$ and $Y$. The random variables $X$ and $Y$ satisfy the stop-loss order, or equivalently the increasing convex order, written as $X \leq_{s l} Y$ (or $X \leq_{i c x} Y$ ), if $\pi_{X}(x) \leq \pi_{Y}(x)$ for all $x$. A sufficient condition for a stop-loss order relation is the dangerousness order relation, written as $X \leq_{D} Y$, defined by the once-crossing condition

$$
\begin{aligned}
& F_{X}(x) \leq F_{Y}(x) \text { for all } x<c, \\
& F_{X}(x) \geq F_{Y}(x) \text { for all } x \geq c,
\end{aligned}
$$

where $c$ is some real number, and the requirement $\mu_{X} \leq \mu_{Y}$ (Lemma 2.1). By equal means $\mu_{X}=\mu_{Y}$, the ordering relations $\leq_{s l}$ and $\leq_{D}$ are precised by writing $\leq_{s l}=$ and $\leq_{D,=}$. The partial stop-loss order by equal means is also called convex order and denoted by $\leq_{c x}$. The probabilistic attractiveness of the partial order relations $\leq_{s t}$ and $\leq_{s l}$ is corroborated by several invariance properties (e.g. Kaas et al. (1994), chap. II.2 and III.2, or Shaked and Shanthikumar (1994)). For example, both of $\leq_{s t}$ and $\leq_{s l}$ are closed under convolution and compounding, and $\leq_{s l}$ is additionally closed under mixing and conditional compound Poisson summing.

In applications, to establish stop-loss order comparison properties, one requires some fundamental facts and equivalent characterizations. First of all, the following well-known elementary equivalent statements hold:

$(S L 1) \quad X \leq_{s l} Y$

(SL2) $\quad E[\varphi(X)] \leq E[\varphi(Y)]$ for all increasing convex functions $\varphi(x)$

(SL3) $\quad E[\max (x, X)] \leq E[\max (x, Y)]$ uniformly for all $x \in R$ 
A famous and widely known sufficient condition for stop-loss order is summarized in the following property.

Lemma 2.1. (Karlin-Novikoff (1963) once-crossing condition, Lemma of Ohlin (1969)). Let $X$ and $Y$ be random variables with distributions $F_{X}(x), F_{Y}(x)$ and suppose that $X \leq_{D} Y$, as defined in (2.2). Then the stop-loss order relation $X \leq_{s l} Y$ is satisfied.

Proof. By assumption, one has the inequalities

$$
\begin{aligned}
& \max (x, X) \leq \max (x, Y), \quad x>c, \\
& \min (x, X) \geq \min (x, Y), \quad x \leq c .
\end{aligned}
$$

In particular, one obtains $E[\max (x, X)] \leq E[\max (x, Y)], x>c$. By $(S L 3)$ above, it remains to show the last inequality for $x \leq c$. This follows immediately from the identity

$$
\max (x, X)=X+x-\min (x, X)
$$

using the assumptions.

A generalized version of the Karlin-Novikoff once-crossing conditions yields the following sign-change characterization of the stop-loss order. Without proof, one finds the relevant conditions in Taylor (1983), which attributes them to Stoyan (1977). However, the previous result by Taylor has not been formulated as a full characterization of stop-loss order.

Theorem 2.1. (Karlin-Novikoff-Stoyan-Taylor crossing conditions for stoploss order). Let $X, Y \in S$ be random variables with means $\mu_{X}, \mu_{Y}$, distributions $F_{X}(x), F_{Y}(x)$ and stop-loss transforms $\pi_{X}(x), \pi_{Y}(x)$. Suppose the distributions cross $n \geq 1$ times in the crossing points $t_{1}<t_{2}<\ldots<t_{n}$. Then one has $X \leq_{s l} Y$ if, and only if, one of the following is fulfilled:

Case 1: The first sign change of the difference $F_{Y}(x)-F_{X}(x)$ occurs from to + , there is an even number of crossing points $n=2 m$, and one has the inequalities

$$
\pi_{X}\left(t_{2 j-1}\right) \leq \pi_{Y}\left(t_{2 j-1}\right), \quad j=1, \ldots, m
$$

Case 2: The first sign change of the difference $F_{Y}(x)-F_{X}(x)$ occurs from + to - , there is an odd number of crossing points $n=2 m+1$, and one has the inequalities

$$
\mu_{X} \leq \mu_{Y}, \quad \pi_{X}\left(t_{2 j}\right) \leq \pi_{Y}\left(t_{2 j}\right), \quad j=1, \ldots, m
$$


Proof. Two cases must be distinguished.

Case 1: The first sign change occurs from - to +

If $X \leq_{s l} Y$, the last sign change occurs from + to - (otherwise $\pi_{X}(x)>\pi_{Y}(x)$ for some $\left.x \geq t_{n}\right)$, hence $n=2 m$ is even. Consider random variables $Z_{0}=Y, Z_{m+1}=X$, and $Z_{j}, j=1, \ldots, m$ with distribution functions

$$
F_{j}(x)= \begin{cases}F_{X}(x), & x \leq t_{2 j-1} \\ F_{Y}(x), & x \geq t_{2 j-1}\end{cases}
$$

For $j=1, \ldots, m$, the Karlin-Novikoff once-crossing condition between $Z_{j+1}$ and $Z_{j}$ is fulfilled with crossing point $t_{2 j}$. A partial integration shows the following mean formulas:

$$
\mu_{j}:=E\left[Z_{j}\right]=\mu_{X}-\pi_{X}\left(t_{2 j-1}\right)+\pi_{Y}\left(t_{2 j-1}\right), \quad j=1, \ldots, m .
$$

Now, by Karlin-Novikoff, one has $Z_{j+1} \leq_{D} Z_{j}, j=1, \ldots, m$, if, and only if, the inequalities $\mu_{j+1} \leq \mu_{j}$ are fulfilled, that is

$$
\begin{aligned}
& \pi_{X}\left(t_{2 j-1}\right)-\pi_{Y}\left(t_{2 j-1}\right) \leq \pi_{X}\left(t_{2 j+1}\right)-\pi_{Y}\left(t_{2 j+1}\right), j=1, \ldots, m-1, \text { and } \\
& \pi_{X}\left(t_{2 m-1}\right)-\pi_{Y}\left(t_{2 m-1}\right) \leq 0,
\end{aligned}
$$

which is equivalent to (2.3). Since obviously $Z_{1} \leq_{s t} Y$, one obtains the ordered sequence

$$
X=Z_{m+1} \leq_{D} Z_{m} \leq_{D} \ldots \leq_{D} Z_{1} \leq_{s t} Z_{0}=Y,
$$

which is valid under (2.3) and implies the result.

Case 2: The first sign change occurs from + to -

If $X \leq_{s l} Y$, then the last sign change occurs from + to - , hence $n=2 m+1$ is odd. Similarly to Case 1 , consider random variables $Z_{0}=Y, Z_{m+1}=X$, and $Z_{j}, j=1, \ldots, m$, with distribution functions

$$
F_{j}(x)= \begin{cases}F_{X}(x), & x \leq t_{2 j} \\ F_{Y}(x), & x \geq t_{2 j}\end{cases}
$$

For $j=0,1, \ldots, m$, the once-crossing condition between $Z_{j+1}$ and $Z_{j}$ is fulfilled with crossing point $t_{2 j+1}$. Using the mean formulas

$$
\mu_{j}:=E\left[Z_{j}\right]=\mu_{X}-\pi_{X}\left(t_{2 j}\right)+\pi_{Y}\left(t_{2 j}\right), \quad j=1, \ldots, m,
$$


the conditions for $Z_{j+1} \leq_{D} Z_{j}$, that is $\mu_{j+1} \leq \mu_{j}, j=0,1, \ldots, m$, are therefore

$$
\begin{aligned}
& \mu_{X}-\mu_{Y} \leq \pi_{X}\left(t_{2}\right)-\pi_{Y}\left(t_{2}\right), \\
& \pi_{X}\left(t_{2 j}\right)-\pi_{Y}\left(t_{2 j}\right) \leq \pi_{X}\left(t_{2 j+2}\right)-\pi_{Y}\left(t_{2 j+2}\right), \quad j=1, \ldots, m-1, \text { and } \\
& \pi_{X}\left(t_{2 m}\right)-\pi_{Y}\left(t_{2 m}\right) \leq 0,
\end{aligned}
$$

which is equivalent to (2.4). One obtains the ordered sequence

$$
X=Z_{m+1} \leq_{D} Z_{m} \leq_{D} \cdots \leq_{D} Z_{1} \leq_{D} Z_{0}=Y,
$$

which is valid under (2.4) and implies the result.

It is instructive to relate this result with another (apparently simpler) known crossing characterization. Instead of crossing points, which describe the sign change properties of the distribution functions, consider slightly more general crossover points, which are defined as follows. A pair $\{\xi, u\}$ of real numbers is a crossover point of the pair $\left\{F_{1}(x), F_{2}(x)\right\}$ of distribution functions if for $i \neq j \in\{1,2\}$ one has

$$
F_{i}\left(\xi^{-}\right) \leq F_{j}\left(\xi^{-}\right) \leq F_{j}(\xi) \leq F_{i}(\xi) \text { and } u=F_{j}(\xi),
$$

or equivalently

$$
F_{i}^{-1}(u) \leq F_{j}^{-1}(u) \leq F_{j}^{-1}\left(u^{+}\right) \leq F_{i}^{-1}\left(u^{+}\right) \text {and } \xi=F_{j}^{-1}(u) .
$$

How are the crossing points related to the crossover points? Clearly, every crossing point is a crossover point. Additionally, there are two crossover points, associated to the end points of the supports of $F_{1}(x), F_{2}(x)$, where no actual sign change between the distributions occurs. Let $\left(a_{i}, b_{i}\right)$, $-\infty \leq a_{i}<b_{i} \leq \infty$, be the open support of $F_{i}(x), i=1,2$, and set $\underline{a}=\min \left\{a_{1}, a_{2}\right\}, \bar{b}=\max \left\{b_{1}, b_{2}\right\}$. Then $(\underline{a}, \bar{b})$ is the open support of the pair $\left\{F_{1}(x), F_{2}(x)\right\}$, and $\{\underline{a}, 0\},\{\bar{b}, 1\}$ are the remaining crossover points. The following characterization has been used by Kertz and Rösler (1992), again without proof.

Corollary 2.1 (Crossover point characterization of the stop-loss order) For $i=1,2$, let $X_{i} \in S$ be random variables with finite means $\mu_{i}$, distributions $F_{i}(x)$, and stop-loss transforms $\pi_{i}(x)$. Then one has $X_{1} \leq_{s l} X_{2}$ if, and only if, for all crossover points $\{\xi, u\}$ of the pair $\left\{F_{1}(x), F_{2}(x)\right\}$, the inequality $\pi_{1}(\xi) \leq \pi_{2}(\xi)$ is fulfilled.

Proof. It suffices to show that the conditions are sufficient. One needs the following additional criteria:

$$
\begin{aligned}
& \pi_{1}(\bar{b}) \leq \pi_{2}(\bar{b}) \Leftrightarrow b_{1} \leq b_{2}, \\
& \pi_{1}(\underline{a}) \leq \pi_{2}(\underline{a}) \Leftrightarrow \mu_{1} \leq \mu_{2} .
\end{aligned}
$$


The first one follows immediately from the integral representation $\pi_{i}(x)=\int_{x}^{\infty} \bar{F}_{i}(t) d t$. For the second one, we distinguish between two cases. If $\underline{a}>-\infty$, then the equivalence follows from the fact that $\pi_{i}(\underline{a})=\mu_{i}-\underline{a}$, $i=1,2$. If $\underline{a}=-\infty$, the inequality

$$
\mu_{1}=\int_{0}^{\infty} \bar{F}_{1}(x) d x-\int_{-\infty}^{0} F_{1}(x) d x \leq \int_{0}^{\infty} \bar{F}_{2}(x) d x-\int_{-\infty}^{0} F_{2}(x) d x=\mu_{2}
$$

can be rearranged to the inequality

$$
\pi_{1}(\underline{a})=\int_{-\infty}^{\infty} \bar{F}_{1}(x) d x \leq \pi_{2}(\underline{a})=\int_{-\infty}^{\infty} \bar{F}_{2}(x) d x,
$$

and vice versa. Since the set $C$ of crossover points equals

$$
C=\{\text { crossing points }\} \cup\{\underline{a}, 0\} \cup\{\bar{b}, 1\},
$$

the inequalities $\pi_{1}(\xi) \leq \pi_{2}(\xi)$ for all $\{\xi, u\} \in C$ imply by the above criteria that the inequalities (2.3) and (2.4) required in Case 1 and Case 2 of the Theorem 2.1 are fulfilled.

The simpler but less precise characterization by crossover points is often sufficient from the theoretical point of view (an example is Theorem 2.3 below). From a practical point of view, Theorem 2.1, together with the ordered sequences (2.8) and (2.12), yields the maximum amount of available information for a stop-loss order relation. In this respect, a detailed application of this result shows that $X_{1} \leq_{s l} X_{2}$ if, and only if, the set $C$ of crossover points is given as follows:

Case 1: $n=2 m$

$$
C=\left\{\left\{a_{1}, 0\right\},\left\{t_{1}, F_{1}\left(t_{1}\right)\right\},\left\{t_{2}, F_{2}\left(t_{2}\right)\right\},\left\{t_{3}, F_{1}\left(t_{3}\right)\right\}, \ldots,\left\{t_{2 m}, F_{2}\left(t_{2 m}\right)\right\},\left\{b_{2}, 1\right\}\right\},
$$

Case 2: $n=2 m+1$

$$
C=\left\{\left\{a_{2}, 0\right\},\left\{t_{1}, F_{2}\left(t_{1}\right)\right\},\left\{t_{2}, F_{1}\left(t_{2}\right)\right\},\left\{t_{3}, F_{2}\left(t_{3}\right)\right\}, \ldots,\left\{t_{2 m+1}, F_{2}\left(t_{2 m+1}\right)\right\},\left\{b_{2}, 1\right\}\right\} .
$$

Some applications, which use the explicit characterization Theorem 2.1, are given in Hürlimann (1998a).

The once-crossing condition of dangerousness order formulated in Lemma 2.1 is not a transitive relation. Though not a proper partial order, it is an important and main tool used to establish stop-loss order between two random variables. In fact, the transitive (stop-loss)-closure of the order $\leq_{D}$, denoted by $\leq_{D^{*}}$, which is defined as the smallest partial order containing all pairs $(X, Y)$ with $X \leq_{D} Y$ as a subset, identifies with the stoploss order. To be precise, $X$ precedes $Y$ in the transitive (stop-loss-)closure of dangerousness, written as $X \leq_{D^{*}} Y$, if there is a sequence of random 
variables $Z_{1}, Z_{2}, Z_{3}, \ldots$, such that $X=Z_{1}, Z_{i} \leq_{D} Z_{i+1}$, and $Z_{i} \rightarrow Y$ in stoploss convergence (equivalent to convergence in distribution plus convergence of the mean). The equivalence of $\leq_{D^{*}}$ and $\leq_{s l}$ is described in detail by Müller (1996) (see also Kaas and Heerwaarden (1992)). In case there are finitely many sign changes between the distributions, the stated result simplifies as follows.

Theorem 2.2. (Dangerousness characterization of stop-loss order) Let $X, Y \in S$ be random variables with finite means such that $X \leq_{s l} Y$. Then there exists a finite sequence of random variables $Z_{1}, Z_{2}, \ldots, Z_{n}$ such that $X=Z_{1}, Y=Z_{n}$ and $Z_{i} \leq_{D} Z_{i+1}$ for all $i=1, \ldots, n-1$.

Proof. This is Kaas et al. (1994), Theorem III.1.3. Alternatively, the ordered sequences (2.8) and (2.12) yield a more detailed constructive proof of this result.

Other characterizations of the stop-loss order can be obtained by transforming the random variables, which must be compared. A simple such result reduces a (degree one) stop-loss order comparison to a degree zero stop-loss order or usual stochastic order comparison by means of the Hardy-Littlewood maximal distribution. For any random variable $X$ with finite mean and quantile function $F_{X}^{-1}(u)$, the Hardy-Littlewood transform $X^{H}$ of $X$ is defined by its quantile function on $[0,1]$ through the formula

$$
\left(F_{X}^{H}\right)^{-1}(u)=\left\{\begin{array}{l}
\frac{1}{1-u} \int_{u}^{1} F_{X}^{-1}(v) d v, \quad u<1 \\
F_{X}^{-1}(1), \quad u=1
\end{array}\right.
$$

Its name stems from the Hardy-Littlewood (1930) maximal function. The random variable $X^{H}$ is the least majorant with respect to $\leq_{s t}$ among all random variables $Y \leq_{s l} X$ (e.g. Meilijson and Nàdas (1979)). Its great importance in applied probability and related fields has been noticed by several further authors, among others Blackwell and Dubins (1963), Dubins and Gilat (1978), Rüschendorf (1991), and Kertz and Rösler (1990/92). A recent actuarial use has been proposed by the author (1998b).

Theorem 2.3. (Reduction of stop-loss order to stochastic order) For $i=1,2$, let $X_{i} \in S$ be random variables with finite means $\mu_{i}$, distributions $F_{i}(X)$, and stop-loss transforms $\pi_{i}(x)$. Then one has $X_{1} \leq_{s l} X_{2}$ if, and only if, one has $X_{1}^{H} \leq_{s t} X_{2}^{H}$.

Proof. (Kertz and Rösler (1992), Lemma 1.8) The basic idea relies on the following geometric property. For each crossover point $\{\xi, u\}$, the identity

$$
\int_{\xi}^{\infty}\left\{F_{1}(t)-F_{2}(t)\right\} d t=\int_{u}^{1}\left\{F_{2}^{-1}(v)-F_{1}^{-1}(v)\right\} d v
$$


expresses the fact that the area between $F_{1}$ and $F_{2}$ to the right of $\xi$ equals the area between $F_{1}^{-1}$ and $F_{2}^{-1}$ to the right of $\mathrm{u}$. From this and the Corollary 2.1 one obtains the result by means of the following equivalences:

$$
\begin{aligned}
& X_{1} \leq_{s l} X_{2} \\
& \Leftrightarrow \pi_{1}(\xi)=\int_{\xi}^{\infty} \bar{F}_{1}(t) d t \leq \int_{\xi}^{\infty} \bar{F}_{2}(t) d t=\pi_{2}(\xi) \text { for all crossover points }\{\xi, u\} \\
& \Leftrightarrow \int_{\xi}^{\infty}\left\{F_{1}(t)-F_{2}(t)\right\} d t \geq 0 \text { for all crossover points }\{\xi, u\} \\
& \Leftrightarrow \int_{u}^{1}\left\{F_{2}^{-1}(v)-F_{1}^{-1}(v)\right\} d v \geq 0 \text { for all crossover points }\{\xi, u\} \\
& \Leftrightarrow\left(F_{1}^{H}\right)^{-1}(u) \leq\left(F_{2}^{H}\right)^{-1}(u) \text { for all } u \in[0,1] \\
& \Leftrightarrow X_{1}^{H} \leq_{s t} X_{2}^{H} .
\end{aligned}
$$

By existence of a common mean $\mu_{1}=\mu_{2}$, the resulting characterization of the convex order $X_{1} \leq_{c x} X_{2} \Leftrightarrow X_{1}^{H} \leq_{s t} X_{2}^{H}$ is found in equivalent form in van der Vecht (1986), p. 69, which attributes the result to D. Gilat. In this situation, there exists also the well-known higher degree stop-loss order reduction property of the integrated tail transform considered by van Heerwaarden (1991), p. 69, whose importance lies in actuarial ruin models (see e.g. Embrechts et al. (1997)). For completeness, one may mention a further characterization of the convex order by means of Markov kernels, which goes back to Blackwell (1953), and still another one by means of fusions for probability measures as studied by Elton and Hill (1992). For this, the interested reader is referred to Szekli (1995).

\section{Properties OF THE DISTORTION PRICING PRINCIPLE}

First, we invalidate S. Wang's proofs of the stop-loss order preserving property $(P 5)$ for the distortion pricing principle through a simple counterexample. Then we focus on a new proof of this important property. For completeness and convenience of the reader, elementary proofs of the other properties $(P 1)-(P 4)$ are also provided, where reference is made to related results in the literature.

\subsection{A diatomic counterexample}

For real numbers $0<a_{2}<a_{1}<b_{1}<b_{2}$ and for $i=1,2$ let $X_{i}$ be a diatomic random variable with support $\left\{a_{i}, b_{i}\right\}$ and probabilities $\left\{p_{i}, 1-p_{i}\right\}, 0<p_{i}<1$, and mean $\mu_{i}=a_{i}+\left(1-p_{i}\right)\left(b_{i}-a_{i}\right)$. Assume $\mu_{1} \leq \mu_{2}$ and $p_{2}<p_{1}$. Then the dangerousness order relation $X_{1} \leq_{D} X_{2}$ (a sufficient condition for $\leq_{s l}$ ) holds 
because $\mu_{1} \leq \mu_{2}$ and the survival functions satisfy the Karlin-Novikoff oncecrossing condition (known as Ohlin's Lemma in actuarial science):

$$
\begin{aligned}
& \bar{F}_{1}(x) \geq \bar{F}_{2}(x), \quad x<c, \\
& \bar{F}_{1}(x) \leq \bar{F}_{2}(x), \quad x \geq c,
\end{aligned}
$$

with $c=a_{1}$. Set $g(x)=x^{\frac{1}{\rho}}, \rho \geq 1$, in (1) to get the $\mathrm{PH}$-transform principle $\Pi_{\rho}[X]=\int_{0}^{\infty} \bar{F}(x)^{\frac{1}{\rho}} d x$. In the notation of Wang, one has

$$
R H S(\rho)=\int_{c}^{\infty}\left\{\bar{F}_{2}(x)^{\frac{1}{p}}-\bar{F}_{1}(x)^{\frac{1}{\rho}}\right\} d x=\left(1-p_{2}\right)^{\frac{1}{\rho}}\left(b_{2}-a_{1}\right)-\left(1-p_{1}\right)^{\frac{1}{\rho}}\left(b_{1}-a_{1}\right) .
$$

Wang (1996b), proof of Theorem 1, states that $R H S(\rho) \geq \bar{F}_{2}\left(a_{1}\right)^{\frac{1}{\rho}-1} \cdot R H S(1)$, or equivalently $\left(1-p_{2}\right)^{\frac{1}{-1}} \geq\left(1-p_{1}\right)^{-\frac{1}{-1}}$. This is not correct because $x^{\frac{1}{\rho}-1}$ is decreasing over $(0, \infty)$ for $\rho>1$ and $\left(1-p_{2}\right)>\left(1-p_{1}\right)$ by assumption. Similarly, Wang (1996a), proof of Theorem 1, states that $R H S(\rho) \geq \bar{F}_{2}\left(a_{1}\right)^{\rho-1} \cdot R H S(1), \quad$ or equivalently $\left(1-p_{2}\right)^{\frac{1}{\rho}-1} \geq\left(1-p_{1}\right)^{\frac{1}{\rho}-1}$, which is false for the same reason. Despite this, one has

$$
\Pi_{\rho}\left[X_{1}\right]=a_{1}+\left(1-p_{1}\right)^{\frac{1}{\rho}}\left(b_{1}-a_{1}\right) \leq a_{2}+\left(1-p_{2}\right)^{\frac{1}{\rho}}\left(b_{2}-a_{1}\right)=\Pi_{\rho}\left[X_{2}\right],
$$

and therefore a correct proof of (P5) must be given.

\subsection{An elementary proof of the stop-loss order preserving property}

In a first step we suppose that $X, Y \in S$. The idea of the proof is simple. For each $X \geq 0$, let $X^{g}$ be the distortion transform with survival function $\bar{F}_{X}^{g}(x)=\bar{g}\left(\vec{F}_{X}(x)\right)$. By Theorem 2.2 it suffices to show that $X \leq_{D} Y$ implies $X^{g} \leq_{s l} Y^{g}$, which in turns implies that $P[X]=E\left[X^{g}\right] \leq E\left[Y^{g}\right]=P[Y]$, hence $(P 5)$. Furthermore, by Theorem 2.3 it suffices to show that $X \leq_{D} Y$ implies $\left(X^{g}\right)^{H} \leq_{s t}\left(Y^{g}\right)^{H}$. (Note that the distributions of $\left(X^{g}\right)^{H}$ and $\left(X^{H}\right)^{g}$ differ in general.)

Suppose that $X \leq_{D} Y$, that is $E[X] \leq E[Y]$ and there exists $q \in(0,1)$ such that

$$
\begin{array}{ll}
F_{X}^{-1}(u) \geq F_{Y}^{-1}(u), & 0 \leq u<q, \\
F_{X}^{-1}(u) \leq F_{Y}^{-1}(u), & q \leq u \leq 1 .
\end{array}
$$

For simplicity, assume that $g(x)$ (resp. $\gamma(x)$ ) is differentiable and has an inverse $g^{-1}(x)$ (resp. $\gamma^{-1}(x)$ ). Then the distortion transform $X^{g}$ has quantile function $\left(F_{X}^{g}\right)^{-1}=\left(\gamma \circ F_{X}\right)^{-1}$, and using (2.14) one obtains for the HardyLittlewood distortion transform $\left(X^{g}\right)^{H}$ the relationships

$$
\left(F_{X}^{g, H}\right)^{-1}(u)=\frac{1}{1-u} \int_{u}^{1}\left(\gamma \circ F_{X}\right)^{-1}(v) d v=\frac{1}{1-u} \int_{\gamma^{-1}(u)}^{1} F_{X}^{-1}(v) d \gamma(v), 0 \leq u<1 .
$$


Similar expressions hold with $X$ replaced by $Y$. One must show that $\left(F_{X}^{g, H}\right)^{-1}(u) \leq\left(F_{Y}^{g, H}\right)^{-1}(u)$ for all $u \in[0,1]$, or equivalently

$$
\int_{w}^{1}\left\{F_{Y}^{-1}(v)-F_{X}^{-1}(v)\right\} d \gamma(v) \geq 0 \text { for all } w \in[0,1] .
$$

If $w \geq q$ this is trivial by the second inequality in (3.2). Let now $0 \leq w<q<1$. Since $\gamma(x)$ is convex, the derivative $\gamma^{\prime}(x)$ is increasing, in particular $\gamma^{\prime}(w) \leq \gamma^{\prime}(q) \leq \gamma^{\prime}(1)$. The affirmation follows from the following chain of equalities and inequalities:

$$
\begin{aligned}
& \int_{w}^{1}\left\{F_{Y}^{-1}(v)-F_{X}^{-1}(v)\right\} d \gamma(v) \\
& =-\int_{w}^{q}\left\{F_{X}^{-1}(v)-F_{Y}^{-1}(v)\right\} \gamma^{\prime}(v) d v+\int_{q}^{1}\left\{F_{Y}^{-1}(v)-F_{X}^{-1}(v)\right\} \gamma^{\prime}(v) d v \\
& \geq \gamma^{\prime}(q) \cdot \int_{w}^{1}\left\{F_{Y}^{-1}(v)-F_{X}^{-1}(v)\right\} d v \geq \gamma^{\prime}(q) \cdot \int_{0}^{1}\left\{F_{Y}^{-1}(v)-F_{Y}^{-1}(v)\right\} d v \\
& =\gamma^{\prime}(q) \cdot\{E[Y]-E[X]\} \geq 0 .
\end{aligned}
$$

This achieves the proof of the stop-loss order preserving property for the distortion pricing principle in case the finite crossing condition (1.3) holds.

In case $X \leq_{s l} Y$ and there are infinitely many crossing points, the equivalence of $\leq_{s l}$ and $\leq_{D^{*}}$ shows that there is a sequence of random variables $Z_{1}, Z_{2}, Z_{3}, \ldots$, such that $X=Z_{1}, Z_{i} \leq_{D} Z_{i+1}$, and $Z_{i} \rightarrow Y$ in stoploss convergence. For each $n \geq 1$ one has $X \leq_{s l} Z_{n}$ by Theorem 2.2 From the preceding first step, one obtains that $P[X] \leq P\left[Z_{n}\right]$. On the other side, the relation $Z_{1} \leq_{D} Z_{i+1}$ implies $\min \left(Z_{i}, d\right) \leq_{D} \min \left(Z_{i+1}, d\right)$ for all $\mathrm{d}$, from which one deduces by the first step that $P\left[\min \left(Z_{m}, d\right)\right] \leq P\left[\min \left(Z_{m}, d\right)\right]$ for all $d$, all $m \geq n$. Using this, the result follows from the inequality

$P\left[Z_{n}\right]=\lim _{d \rightarrow \infty} P\left[\min \left(Z_{n}, d\right] \leq \lim _{d \rightarrow \infty}\left\{\lim _{m \rightarrow \infty} P\left[\min \left(Z_{m}, d\right)\right]\right\}=\lim _{d \rightarrow \infty} P[\min (Y, d)]=P[Y]\right.$.

The first and third equality is a continuity property satisfied by the Choquet integral, and a fortiori by the distortion pricing principle, which is a special case of it (see Denneberg (1994), or Axiom 4, Theorem 1 to 3 in Wang et al. (1997)). The second equality is an application of the dominated convergence theorem, which is allowed for risks with finite support. 


\subsection{Other properties of the distortion pricing principle}

It is now possible to complete and summarize the main properties of the distortion pricing principle. Up to $(P 5)$ an advanced proof of this is in Denneberg (1994), pp. 64 and 71.

Theorem 3.1. (Main properties of the distortion pricing principle) Let $\mathrm{X}$ be a non-negative random variable with survival function $\bar{F}_{x}(x)$, and quantile function $F_{X}^{-1}(u)$. Let $g(x)$ be a differentiable increasing concave function on $[0,1]$ such that $g(0)=0, g(1)=1$. Then the functional $P[X]=\int_{0}^{\infty} g[\bar{F}(x)] d x=\int_{0}^{1} F_{X}^{-1}(u) d \gamma(u)$ with $\gamma(x)=1-g(1-x)$, satisfies the properties $(P 1)-(P 5)$.

Proof. $(P 1)-(P 3)$ are easily shown as follows (see also Denneberg (1990)):

(P1) Since $g(x)$ is increasing concave on $[0,1]$ and $g(0)=0, g(1)=1$, one has $g(x) \geq x$ and therefore $P[X] \geq \int_{0}^{\infty} \bar{F}(x) d x=E[X]$.

(P2) One first shows that $P[X]$ preserves $\leq_{s t}$, which is obvious because $X \leq_{s t} Y$ is equivalent with $F_{X}^{-1}(u) \leq F_{Y}^{-1}(u)$ for all $u \in(0,1)$. Since $X \leq_{s t} Y:=\sup [X]$, the property follows.

(P3) This property follows from the facts $F_{X+b}^{-1}(u)=F_{X}^{-1}(u)+b$ and $F_{a \cdot X}^{-1}(u)=a \cdot F_{X}^{-1}(u)$ for $a \geq 0$.

(P4) That this holds when $\gamma(x)$ has a bounded density is mentioned by Denneberg (1990). Using Wang (1995a), Appendix, one relaxes this condition as follows, where differentiability of $g(x)$ is here not assumed. (The idea of proof is attributed to $\mathrm{O}$. Hesselager). A simple property of concave functions is required.

Lemma 3.1. Let $0<a<b$ and suppose $g(x)$ is concave for $x \geq 0$. Then for any $x \geq 0$ one has the inequality $g(x+b)-g(x+a) \leq g(b)-g(a)$.

Proof. It is well-known that $g(x)$ is concave if, and only if, one has

$$
\frac{g(y)-g(x)}{y-x} \geq \frac{g(z)-g(y)}{z-y} \text { for all } 0 \leq x<y<z .
$$

Two successive applications of this criterion to $a<b \leq x+a<x+b$, respectively $a<x+a<b<x+b$, yields the desired inequality.

It suffices to show (P4) for arbitrary $Y$ and a discrete $X$ taking values in $\{0, \ldots, n\}$. Indeed, applying (P3), the result holds then for $X \in\{k, \ldots, n+k\}$ and $X \in\{k h, \ldots,(n+k) h\}, k \in N_{+}, h>0$ arbitrary. Since any random variable can be approximated closely by a discrete random variable with small enough $h$, the property will hold for arbitrary $X$. One uses mathematical induction. For $n=0$ the affirmation is obvious. To show 
the induction step $n \rightarrow n+1$ for $(X, Y)$ with $X \in\{0, \ldots, n+1\}$, let $\left(X^{\prime}, Y^{\prime}\right)$ be distributed as $(X, Y \mid X>0)$. Since $X^{\prime} \in\{1, \ldots, n+1\}$ the induction hypothesis states that $P\left[X^{\prime}+Y^{\prime}\right] \leq P\left[X^{\prime}\right]+P\left[Y^{\prime}\right]$. With $\varepsilon=\operatorname{Pr}(X=0)$ and $\bar{F}_{Y \mid 0}(x)=\operatorname{Pr}(Y>x \mid U=0)$ one has for $x>0$ :

$$
\begin{aligned}
& \bar{F}_{X}(x)=(1-\varepsilon) \bar{F}_{X^{\prime}}(x), \\
& \bar{F}_{Y}(x)=\varepsilon \bar{F}_{Y \mid 0}(x)+(1-\varepsilon) \bar{F}_{Y^{\prime}}(x), \\
& \bar{F}_{X+Y}(x)=\varepsilon \bar{F}_{Y \mid 0}(x)+(1-\varepsilon) \bar{F}_{X^{\prime}+Y^{\prime}}(x) .
\end{aligned}
$$

According to Lemma 3.1, one obtains for $x>0$ that

$$
\begin{aligned}
& g\left(\bar{F}_{X+Y}(x)\right)-g\left(\bar{F}_{X}(x)\right) g\left(\bar{F}_{Y}(x)\right) \leq g\left((1-\varepsilon) \bar{F}_{X^{\prime}+Y^{\prime}}(x)\right)-g\left((1-\varepsilon) \bar{F}_{X^{\prime}}(x)\right) \\
& -g\left((1-\varepsilon) \bar{F}_{Y^{\prime}}(x)\right) .
\end{aligned}
$$

Observe now that $k(x):=\frac{g((1-\varepsilon) x)}{g(1-\varepsilon)}$ is increasing concave on $[0,1]$ such that $k(0)=0, k(1)=1$. Integrate on both sides of the last inequality and use the induction assumption for the function $k(x)$ to see that

$$
\begin{aligned}
& P[X+Y]-P[X]-P[Y] \\
& \leq g(1-\varepsilon) \cdot\left\{\int_{0}^{\infty} k\left(\bar{F}_{X^{\prime}+Y^{\prime}}(x)\right) d x-\int_{0}^{\infty} k\left(\bar{F}_{X^{\prime}}(x)\right) d x-\int_{0}^{\infty} k\left(\bar{F}_{Y^{\prime}}(x)\right) d x\right\} \leq 0 .
\end{aligned}
$$

This shows $(P 4)$.

Since the property $(P 5)$ has been shown in Section 3.2, the proof is complete.

Note added in proof. At the time this paper has been accepted for publication, the author has received a related paper by Dhaene et al. (1997). These authors present in particular an alternative proof of the stoploss order preserving property of the distortion functional, whose idea is due to A. Müller. Moreover, their Theorem 3 characterizes stop-loss order using the distortion functional in a way dual to the classical characterization (SL1)-(SL3) based on the expected value functional. Finally, the author is grateful to A. Müller for pointing out an error in the elementary proof of Section 3.2.

\section{REFERENCES}

BLACKwell, D. (1953). Equivalent comparisons of experiments. Annals of Mathematical Statistics 24, 265-272.

BLACKWEll, D. and L.E. Dubins (1963). A converse to the dominated convergence theorem. Illinois Journal of Mathematics 7, 508-514.

BühlmanN, H. (1970). Mathematical Methods in Risk Theory. Springer-Verlag. BühlmanN, H. (1980). An economic premium principle. ASTIN Bulletin 11, 52-60.

Büllman,, H. (1984). The general economic premium principle. ASTIN Bulletin 14, 13-21. 
Bühlmann, H., Gagliardi, B., Gerber, H.U. and E. Straub (1977). Some inequalities for stop-loss premiums. ASTIN Bulletin 9, 75-83.

Chateauneuf, A., Kast, R. and A. Lapied (1996). Choquet pricing for financial markets with frictions. Mathematical Finance 6(3), 323-30.

DenNeBerg, D. (1985). Valuation of first moment risk for decision purposes in Finance and Insurance. In: Goppel, H., Henn, R. (ed.) 3. Tagung Geld, Banken und Versicherungen, Karlsruhe, 855-869. Verlag Versicherungswirtschaft, Karlsruhe.

Denneberg, D. (1990). Premium calculation: why standard deviation should be replaced by absolute deviation. ASTIN Bulletin 20, 181-190.

DenneberG, D. (1994). Non-Additive Measure and Integral. Theory and Decision Library, Series B, vol. 27. Kluwer Academic Publishers.

Dhaene, J., Wang, S., Young, V. and M.J. Goovaerts (1997). Comonotonicity and maximal stop-loss premiums. Submitted.

Dubins, L.E. and D. GILAT (1978). On the distribution of the maxima of martingales. Transactions of the American Mathematical Society 68, 337-38.

EltoN, J. and T.P. Hill (1992). Fusions of a probability distribution. The Annals of Probability 20(1), 421-54.

Embrechts, P., Klüppelberg, C. and Th. Mikosch (1997). Modelling Extremal Events for Insurance and Finance. Applications of Mathematics - Stochastic Modelling and Applied Probability, vol. 33.

HARDY, G.H. and J.E. LiTTLEWOoD (1930). A maximal theorem with function-theoretic applications. Acta Mathematica 54, 81-116.

HeerwaArden, van A.E. (1991). Ordering of risks: theory and actuarial applications. Ph.D. Thesis, Tinbergen Research Series no. 20, Amsterdam.

HeerwaARden, van A.E. and R. KaAs (1992). The Dutch premium principle. Insurance: Mathematics and Economics 11, 129-133.

HeilmanN, W.-R. (1987). Grundbegriffe der Risikotheorie. Verlag Versicherungswirtschaft, Karlsruhe. (English translation (1988). Fundamentals of Risk Theory)

HúrimanN, W. (1993). Optimal stop-loss limits under non-expected utility preferences. In Operations Research '92, 550-552. Physica-Verlag.

HURLIMANN, W. (1994). A note on experience rating, reinsurance and premium principles. Insurance: Mathematics and Economics 14, 197-204.

HürLIMANN, W. (1995a). Links between premium principles and reinsurance. International Congress of Actuaries, Brussels, vol. 2, 141-167.

HürlimanN, W. (1995b). Transforming, ordering and rating risks. Bulletin of the Swiss Association of Actuaries, 213-236.

HürimanN, W. (1997). On quasi-mean value principles. Blätter der Deutschen Gesellschaft für Versicherungsmathematik XXIII, Heft 1, 1-16.

HÜRLIMANN, W. (1998a). Truncation transforms, stochastic orders and layer pricing. 26-th International Congress of Actuaries, June 1998, Birmingham.

HúlimanN, W. (1998b). On distribution-free safe layer-additive pricing. Appears in Insurance: Mathematics and Economics.

KaAS, R. and A.E. van HeerwaARden (1992). Stop-loss order, unequal means, and more dangerous distributions. Insurance: Mathematics and Economics 11, 71-77.

KaAs, R., Heerwarden, van A.E. and M.J. Goovaerts (1994). Ordering of Actuarial Risks. CAIRE Education Series 1, Brussels.

Karlin, S. and A. Novikoff (1963). Generalized convex inequalities. Pacific Journal of Mathematics 13, 1251-1279.

KERTZ, R.P. and U. RösLER (1990). Martingales with given maxima and terminal distributions. Israel Journal of Mathematics 69, 173-192.

KERTZ, R.P. and U. RöSLER (1992). Stochastic and convex orders and lattices of probability measures, with a martingale interpretation. Israel Journal of Mathematics 77, 129-164.

Lemaire, J. (1988). Actuarial challenges of reinsurance. Proceedings of the 23-th International Congress of Actuaries, Helsinki.

MeILIJSON, I. and A. NÁdAS (1979). Convex majorization with an application to the length of critical paths. Journal of Applied Probability 16, 671-77. 
MÜLLER, A. (1996). Ordering of risks: a comparative study via loss-stop transforms. Insurance: Mathematics and Economics 17, 215-222.

OHLIN, J. (1969). On a class of measures of dispersion with application to optimal reinsurance. ASTIN Bulletin 5, 249-66.

RüSCHENDORF, L. (1991). On conditional stochastic ordering of distributions. Advances in Applied Probability 23, 46-63.

Shaked, M., Shanthikumar, J.G. (1994). Stochastic orders and their applications. Academic Press, New York.

STOYAN, D. (1977). Qualitative Eigenschaften und Abschätzungen Stochastischer Modelle. Akademie-Verlag, Berlin. (English version (1983). Comparison Methods for Queues and Other Stochastic Models. J. Wiley, New York.)

SzekLI, R. (1995). Stochastic Ordering and Dependence in Applied Probability. Lecture Notes in Statistics 97. Springer-Verlag.

TAYLOR, J.M. (1983). Comparisons of certain distribution functions. Math. Operationsforschung und Statistik, Ser. Stat. 14(3), 397-408.

VAN DER VECHT, D.P. (1986). Inequalities for stopped Brownian motion. C.W.I. Tracts 21, Mathematisch Centrum, Amsterdam.

WANG, S. (1995a). Insurance pricing and increased limits ratemaking by proportional hazards transforms. Insurance: Mathematics and Economics 17, 43-54.

WANG, S. (1995b). Insurance pricing and increased limits ratemaking by proportional hazards transforms. Proc. XXV. Int. Congress of Actuaries Brussels, vol. 2, 293-323.

WANG, S. (1995c). Riks loads on life/non-life insurance: a unified approach. XXVI. ASTIN Colloquium, Leuven.

WANG, S. (1996a). Premium calculation by transforming the layer premium density. ASTIN Bulletin 26, 71-92.

WANG, S. (1996b). Ordering of risks under PH-transforms. Insurance: Mathematics and Economics 18, 109-114.

WANG, S., V.R. Young and H.H. PANJer (1997). Axiomatic characterization of insurance prices. Insurance: Mathematics and Economics 21, 173-183.

\section{WERNER HÜRLIMANN \\ Mathematik KB L \\ "Winterthur" \\ Paulstr. 9 \\ CH-8401 Winterthur}

Switzerland 